\title{
Use of Electrochemical Tests for Assessment of the Effect of Erosive Particle Size on the Erosion-Corrosion Behaviour of AISI 304L Austenitic Stainless Steel
}

\author{
Diana Maria López ${ }^{a}$, Neusa Alonso Falleiros ${ }^{b}$, André Paulo Tschiptschin ${ }^{b *}$ \\ ${ }^{a}$ Departamento de Ingeniería Mecánica, Universidad Nacional de Colombia - UNAL, \\ Medellin, Colombia \\ ${ }^{b}$ Metallurgical and Materials Engineering Department, University of São Paulo - USP, SP, Brazil
}

Received: March 23, 2015; Revised: November 21, 2015; Accepted: January 26, 2016

\begin{abstract}
The degradation of AISI 304L stainless steel under static corrosion, liquid impingement and corrosion-erosion was studied using polarization curves. Static corrosion and liquid impingement tests were performed in $3.5 \% \mathrm{NaCl}$ solution. Corrosion-erosion tests were performed in the same solution, containing $10 \mathrm{wt}-\%$ of three different $\mathrm{SiO}_{2}$ particle sizes. Synergism between erosion and corrosion was correlated to shifts in the polarization curves. The passive current densities increased and the pitting potential decreased for liquid impingement and with addition of $\mathrm{SiO}_{2}$ particles to the electrolyte. Increasing the particle size led to a greater increase in the current densities, although the pitting potential did not significantly changed. The effects of erosion on corrosion are discussed in terms of the passive film breakdown and reformation.
\end{abstract}

Keywords: Erosion-corrosion synergism, Austenitic stainless steel, Electrochemical techniques, Polarization curves

\section{Introduction}

Corrosion-erosion (CE) is present in many industrial systems such as centrifugal pumps, pipeline accessories and elements for handling slurries, especially for applications in aggressive oilfield and marine environments requiring outstanding corrosion resistance and mechanical properties. During last decades, several studies have been developed to achieve a better knowledge of corrosion-erosion interactions in these systems ${ }^{1-7}$. Wear and corrosion processes involve complex mechanical and electrochemical mechanisms, the combined actions of which often result in a significant increase in material degradation. This increase is called synergism and it is frequently found in passive alloys such as stainless steels. Erosion affects corrosion and corrosion affects erosion, changing the kinetics of both separate processes. The mechanism responsible for this synergism is related with the additional difficulty to repair the passive film after it has been damaged under the impingement of the slurry and the formation of lips and highly deformed zones that can be easily corroded. Different methods have been used to estimate the synergism between corrosion and wear. Watson et al. ${ }^{8}$ list some of them: wear track volume or mass loss, penetration rates, and size of current intensity during wear. In the classical approach, the synergism is defined as the difference between the total corrosion-wear mass loss and the sum of the corrosion and wear mass losses, measured separately. Electrochemical techniques have also been used to assess the synergism between erosion and corrosion. Madsen ${ }^{9}$ quantified the synergism through mass loss measurements and introduced the use of potentiodynamic tests for corrosion-erosion analysis. In the

*e-mail: antschip@usp.br same year, Guenbour et al. ${ }^{10}$ published a paper where the corrosion-erosion resistance of different stainless steels in a chloride solution and in sulphuric acid is studied. They used potentiodynamic polarization curves to study the effect of $\mathrm{SiC}$ particle additions on the corrosion behaviour. Since then, new electrochemical techniques have been used, trying to understand the mechanisms behind the corrosion-erosion synergism. Open Circuit Potential Measurements, Scanning Electrochemical Microscopy, Ellipsometry, Electrochemical Impedance Spectroscopy, and Electrochemical Noise Methods have been reported ${ }^{11-15}$. Most of these previous works agree in that the introduction of erosive particles turns the surface more active, shifts the corrosion potential to lower values and promotes an increase of current densities, in comparison with the results obtained without particles.

Electrochemical measurements can give significant information about wear assisted corrosion mechanisms, because the electrochemical state of the surfaces is highly dependent on these conditions. In particular, potentiodynamic polarization techniques allow obtaining plots of current as a function of applied potential. From this information it is possible to determine the effect of wear on the active/passive behaviour of materials at different potentials and evaluate changes in the kinetics which controls the rate of corrosion ${ }^{16}$.

A limited number of works have treated the variation of flow velocity, particle size or impact angle on the corrosion-erosion resistance of stainless steels using electrochemical techniques. Zheng et al. ${ }^{17}$ studied the effect of impact velocity on the transition from passivation to erosion-corrosion of an AISI 304 stainless steel in sand-containing $\mathrm{NaCl}$ solution and established $10 \mathrm{~m} / \mathrm{s}$ as a critical flow velocity. Their results 
showed that at a velocity below the critical flow velocity, the open-circuit potential shifts in the positive direction whereas it shifts to more negative values when the flow velocity is above the critical flow velocity. Rajahram et al. ${ }^{18}$ considered the effect of velocity in an AISI 316 tested under erosion-corrosion with $3.5 \% \mathrm{NaCl}$ and $40^{\circ} \mathrm{C}$. At the open circuit potential, they found a continuous increment in the current value when velocity is raised. This was attributed to the increase in kinetic energy of the particle and the mass transport in the fluid flow. Lu et al. ${ }^{19}$ tested an AISI 304 stainless steel in tap water and in a single-impact erosion test, using an impact angle of $45^{\circ}$. They reported that the current density is almost independent of impact velocity between 5 and $15 \mathrm{~m} / \mathrm{s}$, suggesting that the repassivation of the eroded surface is unaffected by the mass transfer in the boundary layer. The comparison of these results is difficult, since the testing conditions are different, as they suggest that little changes in test parameters can considerably change the results.

Rajahram et al. ${ }^{18}$ also studied the effect of particle size, testing three groups of particle sizes: 90-150, 150-300 and $300-600 \mathrm{~mm}$. They observed the highest corrosion currents for the medium sized particles, followed by coarse particles and the lowest current levels for the fine particles. They proposed that for a constant particle weight concentration, a balance between number and damage related to the size of particle is established. Meanwhile, Lu et al. ${ }^{19}$ also studied the effect of the impact angle at $10 \mathrm{~m} / \mathrm{s}$ and found that the current response and damaged area reach their maximums at an impact angle around $30^{\circ}$. Finally, Zhao et al. ${ }^{20}$ studied the effect of $20^{\circ}$, $60^{\circ}$ and $75^{\circ}$ impact angle on the electrochemical response of AISI 316 stainless steel samples submitted to erosion in $3.5 \% \mathrm{NaCl}$ at $20 \mathrm{~m} / \mathrm{s}$ and $45^{\circ} \mathrm{C}$, using polarization curves and electrochemical impedance spectroscopy. They proposed that the anodic current densities for the specimens tested with $20^{\circ}$ and $75^{\circ}$ impact angles are larger than the one with impact angle of $60^{\circ}$. They propose that it is probably due to the dissolution of the metal at the small holes or cracks caused by the erosion. In addition, corrosion potential decreases when the angle increases, but there is little effect of impact angle on pitting potential.

In a previous work ${ }^{21}$ the authors studied the effect of introducing nitrogen on the corrosion-erosion response of AISI 304 and AISI 410 stainless steels, testing at $8 \mathrm{~m} / \mathrm{s}$, $90^{\circ}$ of impact angle and a fixed particle size. It was found a clear effect of nitrogen on the corrosion-erosion synergism of martensitic steel, reducing it by $20 \%$. The effect was not so noticeable in the austenitic steel. However, the pitting potential was significantly improved in both steels with the introduction of nitrogen.

In this work the degradation mechanisms of an AISI 304L stainless steels under static corrosion, liquid impingement and corrosion-erosion conditions were studied using polarization curves. The effects of particle size and impact angle were considered. The synergism between erosion and corrosion was evaluated by measuring the shift in the passive current density and corrosion potential for different conditions. The electrochemical parameters of the passive regime and pitting attack were related to flow conditions as well as to the mechanical properties of the surfaces.

\section{Experimental procedure}

\subsection{Materials}

Austenitic AISI 304L stainless steel samples were solution-annealed during 1 hour at $1373 \mathrm{~K}$ in an Ar atmosphere and water quenched. The microstructure was constituted solely of austenite grains with grain size around $40 \mu \mathrm{m}$. Grain size was determined using the average grain intercept, according to ASTM E112 and Figure 1 shows the microstructure of the

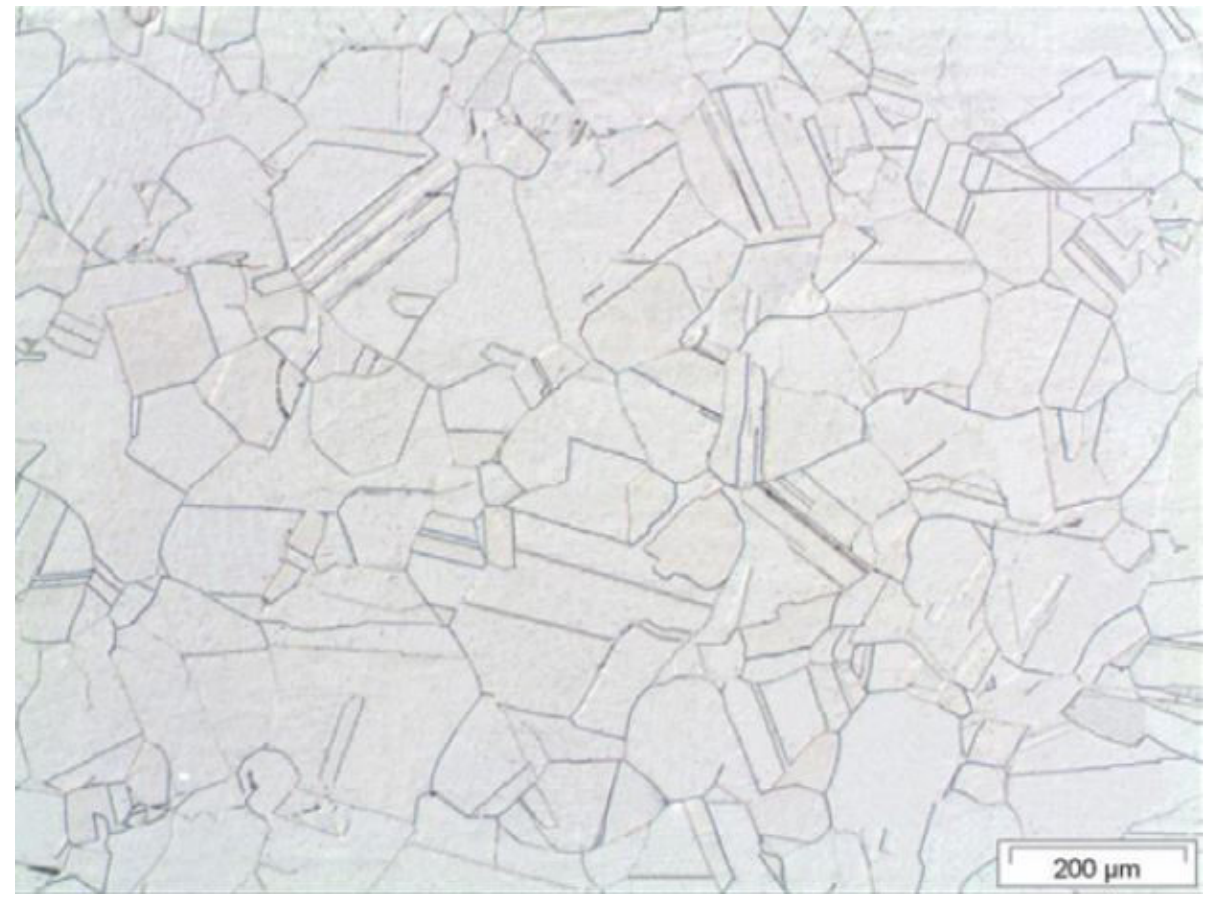

Figure 1. Microstructure of the solution-annealed AISI 304L stainless steel. Average grain size $40 \mu \mathrm{m}$. 
solution-annealed alloy. The chemical composition and the hardness of the alloy are shown in Table 1.

\subsection{Slurry Erosion and Electrochemical tests}

Polarization curves for AISI 304L specimens were obtained in a $E G \& G$ Princeton Applied Research (PAR) 273 potentiostat with an $\mathrm{Ag} / \mathrm{AgCl}$ reference electrode and platinum counter-electrode. The samples were polished in emery paper up to grade 600, ultrasonically cleaned, rinsed and dried with air before the tests. A potential scan rate of $1 \mathrm{mV} / \mathrm{s}$ was used, with starting point at $100 \mathrm{mV}$ under the corrosion potential.

Once the corrosion potential was stabilized to less than a programmed $3 \mathrm{mV}$ "drift rate", five minutes were set to start the measurement. For times longer than five minutes crevice corrosion could start, leading to loss of the electrochemical test.

The Pitting Potential (Ep) and Passive Current Density (Ipass) were computed from the polarization data of three curves for each kind of test. Ep was determined as the potential in which the current density began to increase sharply in the passive region and Ipass was defined as the mean current density in the passive window. Two kinds of experiments were carried out in a slurry wear testing machine described elsewhere ${ }^{21}$ liquid impingement tests were performed in $3.5 \% \mathrm{NaCl}$ solution with a $\mathrm{pH}$ value of 5.1 , while the corrosion-erosion experiments were carried out in the same solution with the addition of $10 \mathrm{wt}-\% \mathrm{SiO}_{2}$ particles. Three different particle sizes were used: 50-75 AFS, with mean size between 200 and $300 \mu \mathrm{m}, 40-50$ AFS, with mean size between 300 and $420 \mu \mathrm{m}$ and 35-40 AFS, with mean size between 420 and $500 \mu \mathrm{m}$. The particle size classification was established using a HMK Sieve shaker.

Figure 2 illustrates the shape and size of the $\mathrm{SiO}_{2}$ particles and the particle sizes. All the samples were immersed in the electrolyte for $5 \mathrm{~min}$ before starting the potential scan. The mean impact velocity of the flow was held around $6.5 \mathrm{~m} / \mathrm{s}$ and impact angle was normal to the surface. Testing time was around 25 and 40 minutes, depending on the behavior of the corrosion potential and pitting potential parameters. Figure 3 shows the home built experimental setup used for the tests. All the measurements were repeated three

Table 1. Chemical composition (wt- $\%$ ) and hardness

\begin{tabular}{ccccccccc}
\hline C & Cr & Ni & Mo & Mn & P & Si & Hardness \\
(HV) & & & & & \\
\hline 0.014 & 18.2 & 8.1 & 0.093 & 1.355 & 0.036 & 0.4 & 0.002 \\
\hline
\end{tabular}

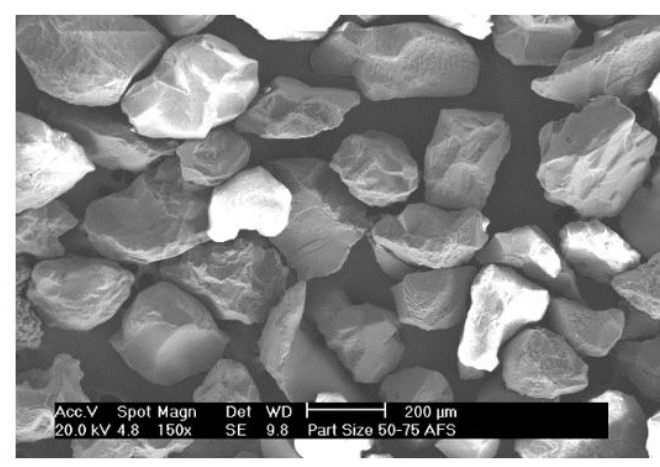

(a)

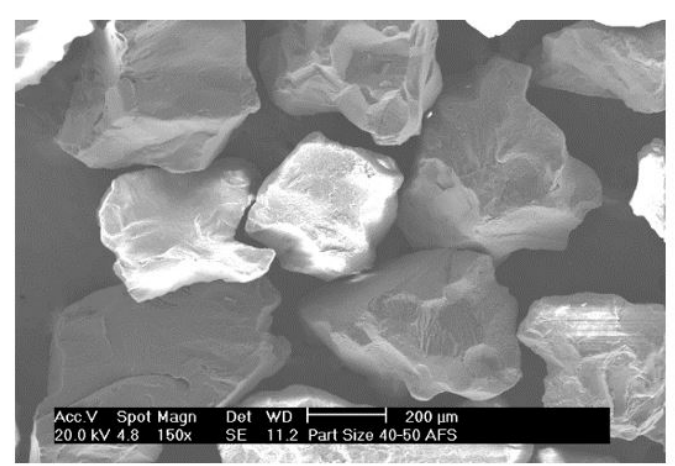

(b)

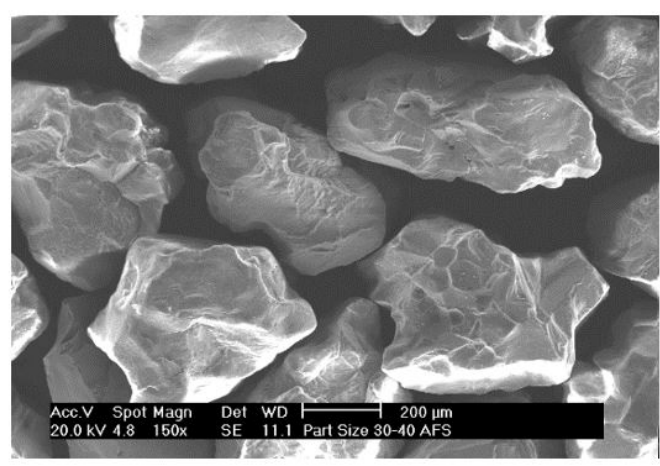

(c)

Figure 2. $\mathrm{SiO}_{2}$ particles used in the corrosion-erosion test. Three different sizes where used: (a) 50-75 AFS (200 - 300 $\left.\mu \mathrm{m}\right)$, (b) 40-50 AFS, $(300-420 \mu \mathrm{m})$ and (c) $35-40 \mathrm{AFS},(420$ and $500 \mu \mathrm{m})$. 

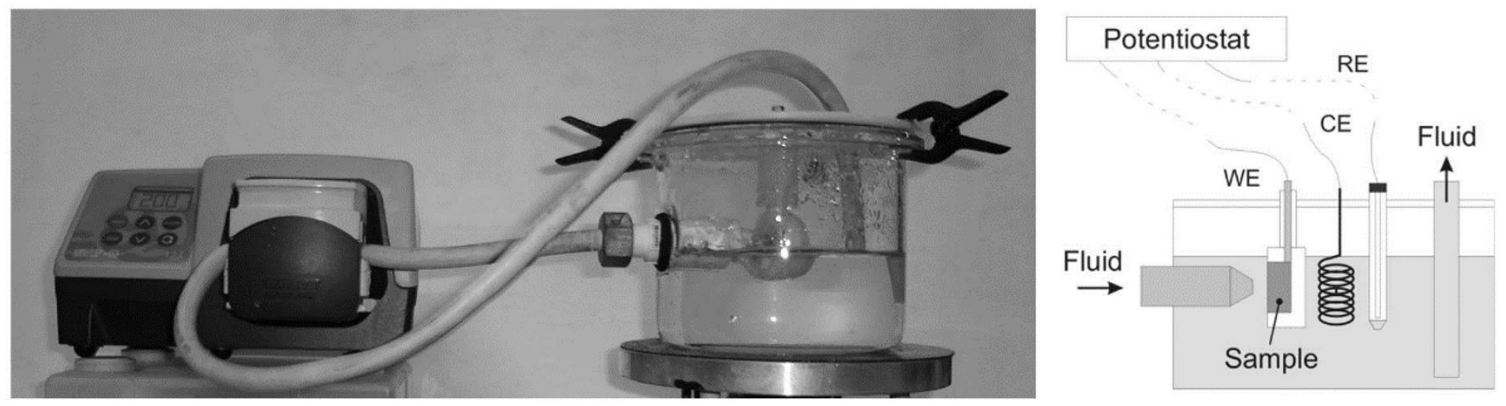

Figure 3. Experimental setup for liquid impingement and corrosion-erosion tests.

times, and the obtained results were reproducible. Small differences between curves were seen, but they did not affect the reproducibility of experiments.

Impact angles of $30^{\circ}$ and $90^{\circ}$ and impact velocity circa $6.5 \mathrm{~ms}^{-1}$ were used; the slurry temperature varied between 298 and $300 \mathrm{~K}$ for all the tests. It is worth noticing that the reported impact angles represent mean values, given the fact that in slurry erosion the particles are considerably deviated when they reach the surface, due to boundary layer and viscosity effects associated to the liquid carrier ${ }^{18}$.

\subsection{Surface Examination and Microstructure Analysis}

The microstructure and surface morphology of the specimens were analyzed by optical $(\mathrm{OM})$ and scanning electron microscopy (SEM), in an OLYMPUS BX60M and a Philips XL30 TMP microscope, respectively.

\section{Results and Discussion}

\subsection{Effect of liquid impingement and erosion-corrosion}

Figure 4 shows potentiodynamic curves obtained for AISI 304L steel in static condition, liquid impingement and erosion-corrosion tests with impact angle of $90^{\circ}$. The curves show a shift to higher current densities when the electrolyte is in movement and when particles are added to the flow. It is noticeable too that both corrosion and pitting potential decrease.

The curve under liquid impingement condition shifts to higher current densities in comparison with the curve obtained under static conditions, demonstrating that corrosion is strongly dependent on mass transfer conditions. The flow of the electrolyte enhances the mass transfer of metallic ions, hindering the formation of the passive layer, and leading to an increase in corrosion and passive current densities. In addition, turbulence within the electrolyte could promote the dissolution and detaching of the passive film. Thinning of the passive layer is basically controlled by the mass transfer rate and by the film growth kinetics, whereas the lateral spreading effect of the jet when it impacts against the solid surface, can produce shear stresses high enough to damage the passive layer ${ }^{11-22}$. At the same time, under these conditions, $\mathrm{E}_{\mathrm{p}}$ decreases due to the growth of a passive layer with poor properties. These results are in accordance

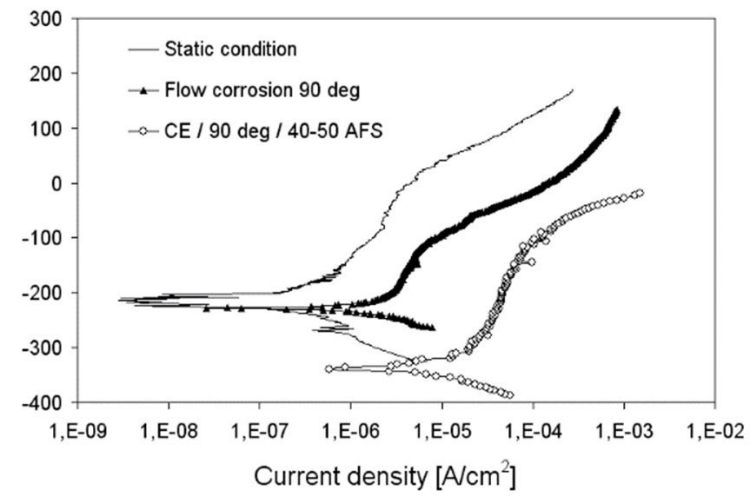

Figure 4. Potentiodynamic curves obtained for AISI 304L steel in static condition and during liquid impingement and erosion-corrosion tests with $90^{\circ}, 6.5 \mathrm{~m} / \mathrm{s}$, and $3.5 \% \mathrm{NaCl}$.

with a previously published one ${ }^{21}$, testing high nitrogen austenitic and martensitic stainless steels, using AISI 304 steel for comparison.

On the other hand, when particles are introduced, new mechanical stresses arise on the surface, damaging continuous and locally the passive layer. This process keeps happening, increasing even more the corrosion and the passive current density, not only because bare material is exposed to the aggressive solution but also due to differences in electrochemical potential between unprotected and protected zones on the surface. As a consequence, the corrosion potential, pitting potential and polarization resistance decrease with the weakening of the passive layer. The values of the electrochemical parameters obtained from the polarization data are listed in Table 2.

SEM images of the damaged surfaces obtained in different conditions are shown in the next figures. The surface of a sample tested in liquid impingement corrosion condition is shown in Figure 5. It is possible to observe a lot of pits distributed on the surface. On the other hand, the corroded-eroded surface is shown in Figure 6. At the center of the scar (Figure 6a), one can see evidences of the erodent particles producing overlapping impacts, causing plastic deformation, whereas the outer region reveals the microcutting action of the particles on the surface (Figure 6b). The cutting marks are associated with particles impinging the surface at an oblique angle due to the hydrodynamic streamlines of the 
Table 2. Electrochemical parameters for different test conditions

\begin{tabular}{|c|c|c|c|c|c|}
\hline Condition & Impact Angle & Particle Size $(\mu \mathrm{m})$ & $\operatorname{Ecorr}(\mathrm{V})$ & $\operatorname{Ep}(V)$ & Rp (Ohms) \\
\hline Static & - & - & -0.223 & 0.032 & 42030 \\
\hline Flow corrosion & 90 & - & -0.229 & -0.098 & 5988 \\
\hline Flow corrosion & 30 & - & -0.289 & -0.082 & 6047 \\
\hline \multirow[t]{3}{*}{ Corrosion Erosion } & & $300-200$ & -0.323 & -0.098 & 1709 \\
\hline & 90 & $420-300$ & -0.338 & -0.100 & 1547 \\
\hline & & $500-420$ & -0.287 & -0.110 & 444 \\
\hline Corrosion Erosion & 30 & $300-200$ & -0.281 & -0.108 & 1531 \\
\hline
\end{tabular}

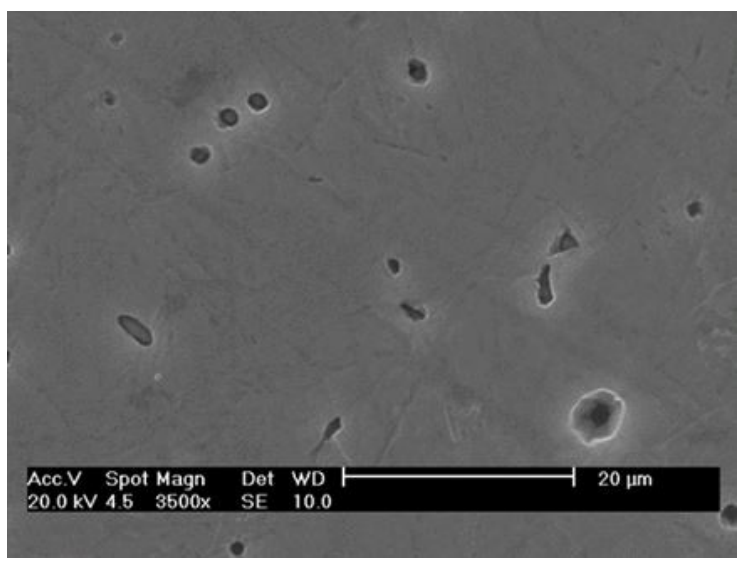

Figure 5. Image of sample surface after liquid impingement corrosion test.

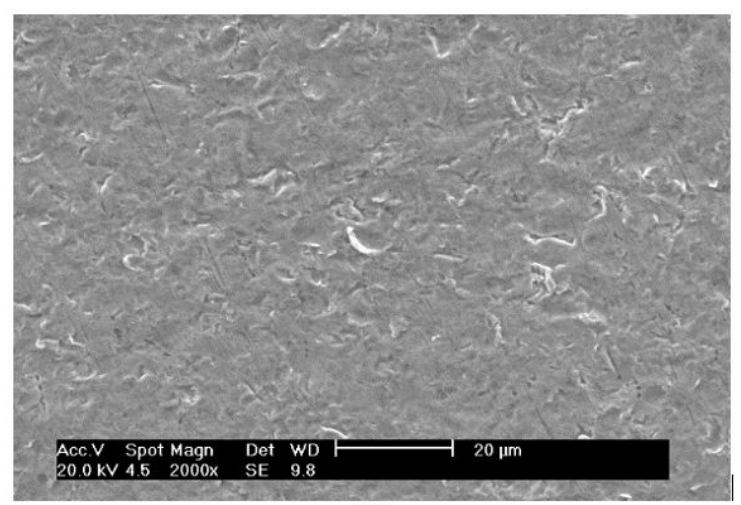

(a)

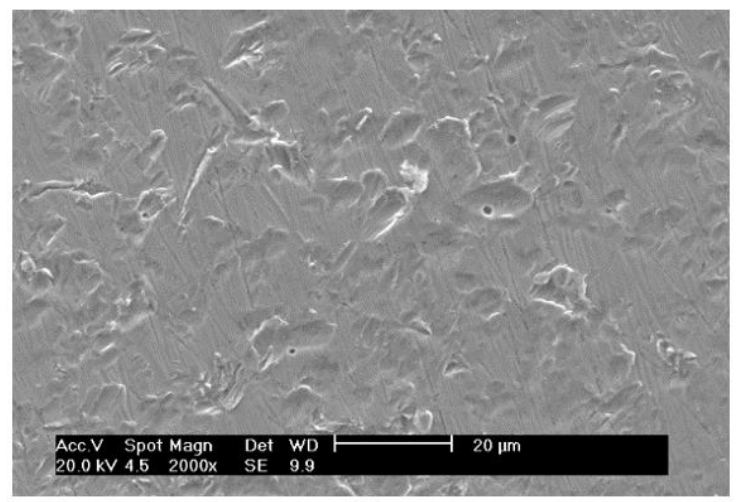

(b)

Figure 6. Aspect of typical erosion marks obtained on the surface of the samples tested under corrosion-erosion with $90^{\circ}$ (a) central region of the scar, (b) outer region. slurry jet, instead of the nominal normal angle between the nozzle of the equipment and the surface of the sample, as illustrated in Figure 7.

\subsection{Effect of particle size}

Figure 8 shows the particle size effect on the polarization curves under corrosion-erosion conditions for an impact angle of $90^{\circ}$.

Increasing the particle size shifts the polarization curves to higher current densities: the higher the particle size the higher the passive current densities and the lower is the polarization resistance (Table 2). Introducing smaller size particles produced almost the same shift of the polarization curves obtained when testing under liquid impingement condition. This behaviour could be related to a retardation effect of particles immediately before the impact, in the region of the boundary layer. This effect is more pronounced when testing with small particles. On the other hand, all the curves obtained under the action of erosive particles showed a low and similar pitting potential, indicating that although the introduction of particles decreased the pitting potential in all cases, the particle size did not seem to have a marked influence on this potential.

Figure 9 shows the damaged surfaces tested with different particle sizes. The surface tested with the smaller particles shows few marks distributed all over the surface (Figure 9a). The indentations are circa $4 \mu \mathrm{m}$ in diameter, small compared to the $200 \mu \mathrm{m}$ average size of the erosive particles, indicating a shallow indenting effect. Figure $9 \mathrm{~b}$ and $\mathrm{c}$ show the tested surfaces with the 300-420 $\mu \mathrm{m}$ (40-50 AFS) and 420-500 $\mu \mathrm{m}$ (35-40 AFS) particles, respectively. When the particle size increases, the number and the size of the marks increase and the surface starts to be covered by these marks until completely erasing the emery paper marks left during grinding.

\subsection{Effect of impact angle}

Figure 10 shows a comparison between some potentiodynamic curves obtained for AISI 304L steel in static condition, liquid impingement and erosion-corrosion tests with 30 and $90^{\circ}$ angles of incidence. The curves obtained with two different particle sizes are shown as well. The potentiodynamic curves obtained for the static, liquid impingement and erosion-corrosion conditions are quite similar to those obtained in a previously published paper, reporting a similar behavior for an AISI 304 austenitic stainless steel ${ }^{21}$. There are no clear differences between the polarization curves when the impact angle is modified; although in the corrosion-erosion curves with the coarse particles, the passive current increases slightly with $30^{\circ}$ in comparison with the normal incidence. However, the observation of the surfaces seems to indicate that particles 


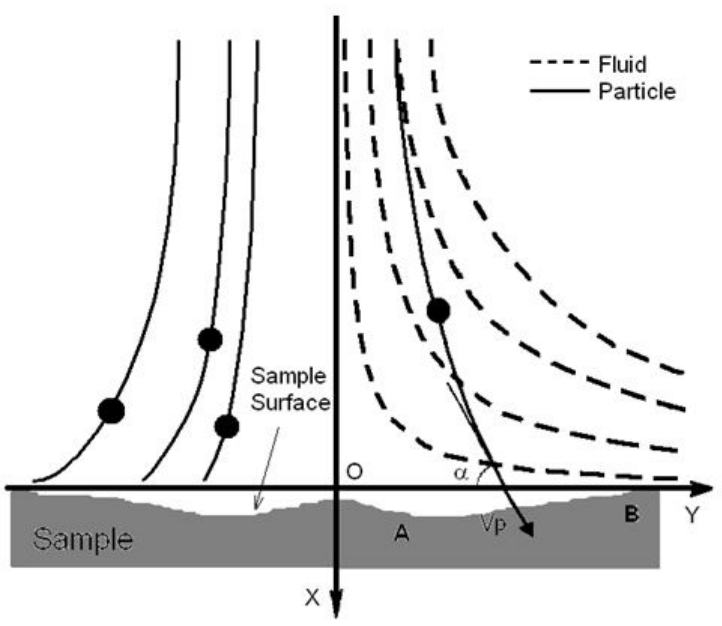

Figure 7. Scheme of a sample surface section during a normal impact, showing the scar where SEM images of figure 5 were taken: (A) at the location of maximum erosion depth within the scar and (B) outside the scar region. Adapted from ${ }^{18}$.

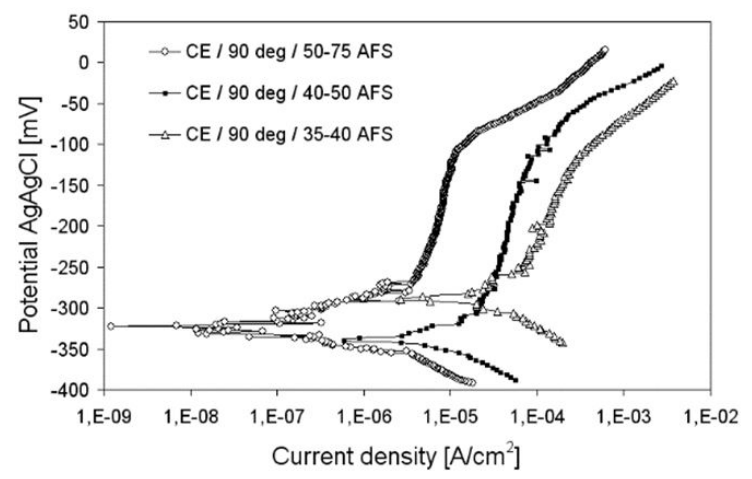

Figure 8. Potentiodynamic curves obtained for AISI 304L steel in erosion-corrosion tests with $3.5 \% \mathrm{NaCl}$ solution, $90^{\circ}, 6.5 \mathrm{~m} / \mathrm{s}$ and different particle size.

impacting with grazing incidence angle remove more material from the surface of the sample when compared to the normal impact. This hypothesis is supported by the observation of a huge number of lips that are nearly detached from the surface during grazing impact. On the other hand, indentation-like marks observed on the surfaces tested with normal impact cause plastic deformation but do not remove a lot of material. This results could be consequence of the low velocity used in this test, but also due to the fact that the potentiodynamic technique can reveal that the passive layer is being removed and that bare material is being exposed, but it does not recognize how much of the substrate material is being removed. This result indicates the need of analyzing the surface damage measuring mass loss, besides the information obtained using polarization curves. This information could clarify the hypothesis about the angle impact effect. Figure 11 shows a comparison of a surface tested in 30 and $90^{\circ}$. Oblique impacts cause the

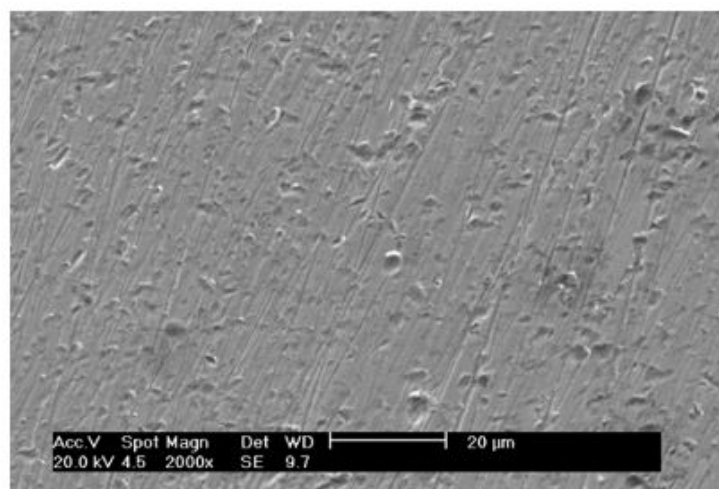

(a)

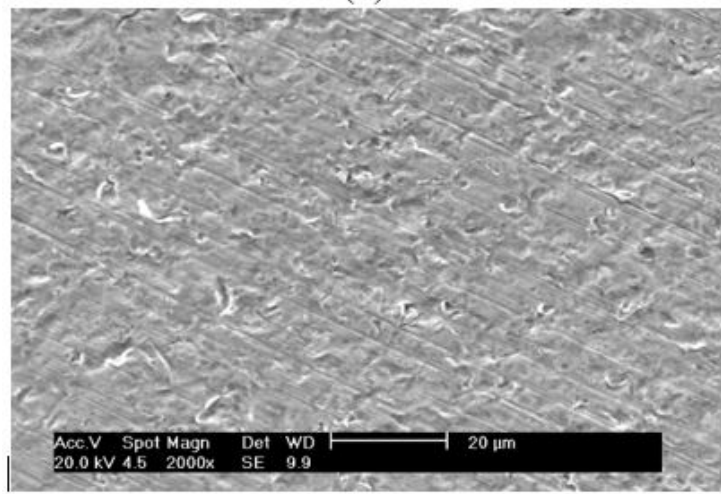

(b)

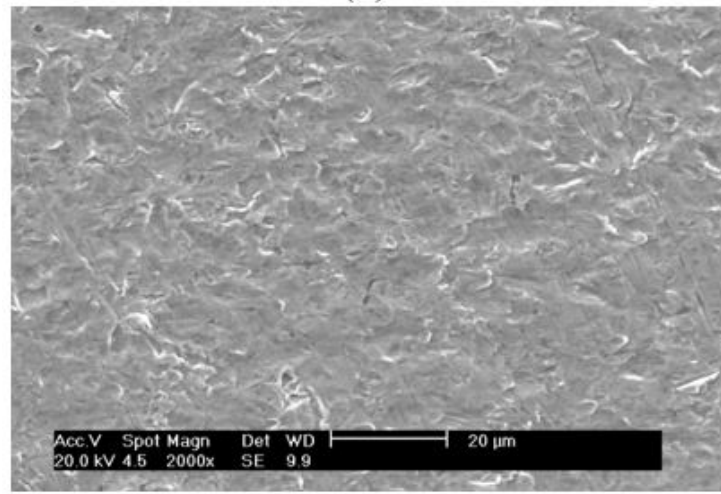

(c)

Figure 9. Surfaces of the samples tested with different grain sizes. (a) fine sand (50-75 AFS), (b) medium sand (40-50 AFS) and (c) coarse sand (35-40 AFS).

formation of grooves and prows, while $90^{\circ}$ incident particles lead to crater formation.

Comparing the electrochemical parameters in Table 2 with those obtained in the previous work ${ }^{21}$, one can see that the variation of impact angle did not significantly changed the pitting potential and polarization resistance over the static condition values obtained in both studies. On the other hand, the effect of quartz particles size could be more thoroughly detailed, as the polarization resistance decreased much more for larger quartz particle sizes. The variation of corrosion potential and pitting potential, resulted quite the same to those reported in the previous paper, leading to the conclusion that the difference in carbon content of the two 


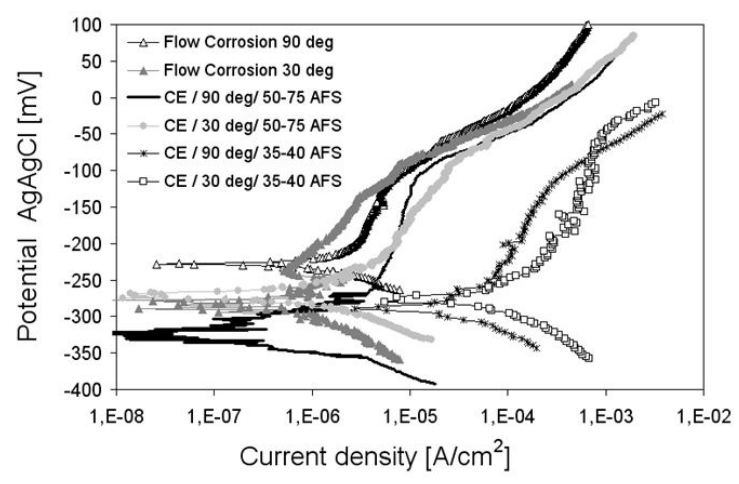

Figure 10. Potentiodynamic curves obtained for AISI 304L steel during liquid impingement and erosion-corrosion tests with $90^{\circ}$ and $30^{\circ}$ in a $3.5 \% \mathrm{NaCl}$ solution and slurries with two different particle sizes.

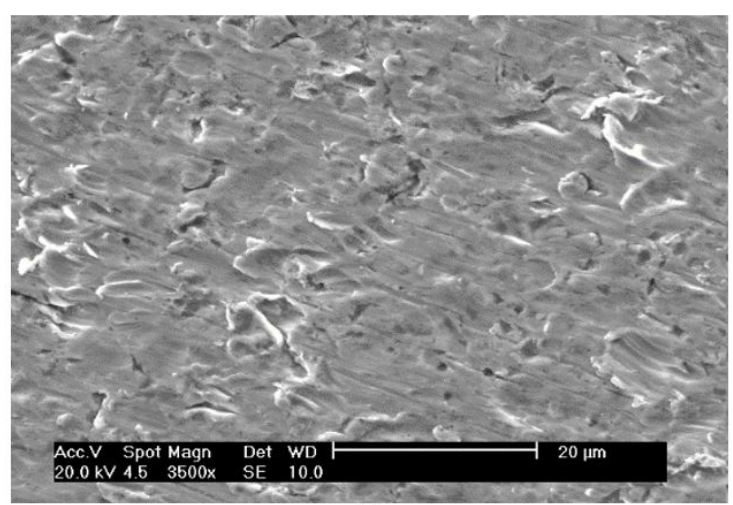

(a)

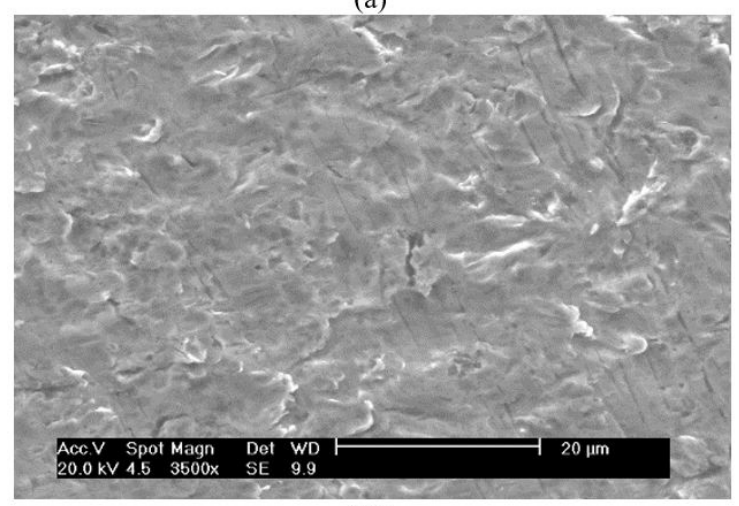

(b)

Figure 11. Comparison of surfaces tested with impact angles of (a) $30^{\circ}$ and (b) $90^{\circ}$.

steels did not affect significantly the flow corrosion and erosion-corrosion, in the conditions studied in both papers.

\subsection{Effect of erosion on the corrosion behaviour:}

As already discussed, the passive current increased significantly by breakdown of the passive film due to the impact of particles and the subsequent reformation of the film. The influence of particle erosion on corrosion is presented in Table 3 for the three particle sizes studied in this work and for normal impact. One can see, in Table 3
Table 3. $\Omega$ parameter to quantify the effect of erosion on corrosion

\begin{tabular}{|c|c|c|c|}
\hline Condition & $\begin{array}{c}\text { Particle Size } \\
(\mu \mathrm{m})\end{array}$ & $\begin{array}{c}\text { Ipass } \\
{\left[\mathbf{A} / \mathbf{c m}^{2}\right]}\end{array}$ & $\Omega$ \\
\hline $\begin{array}{c}\text { Flow } \\
\text { corrosion }\end{array}$ & - & $3.93 \times 10^{-6}$ & \\
\hline \multirow{3}{*}{$\begin{array}{l}\text { Corrosion } \\
\text { Erosion }\end{array}$} & $\begin{array}{c}300-200 \\
(50-75 \text { AFS })\end{array}$ & $6.76 \times 10^{-6}$ & 1.72 \\
\hline & $\begin{array}{c}420-300 \\
(40-50 \text { AFS })\end{array}$ & $4.51 \times 10^{-5}$ & 11.47 \\
\hline & $\begin{array}{c}500-420 \\
(35+40 \text { AFS })\end{array}$ & $1.42 \times 10^{-4}$ & 36.13 \\
\hline
\end{tabular}

that increasing particle size increases steadily the passive current and decreases the ratio between the passive current density, under corrosion-erosion conditions and under liquid impingement:

$$
\Omega=\frac{i_{\text {pass }}^{C E}}{i_{\text {pass }}^{\text {Liq imp }}}
$$

The results obtained represent the behaviour described before, in which the increase of the particle size led to an increase in the effect of the erosion on the corrosion. This parameter allows quantifying and establishing a comparison among different damage levels of the passive layer. The value of the passive current density used to calculate the $\Omega$ parameter was assessed taking the intermediate value of current density of the passive region. Increasing particle size increases the passive current density under erosion-corrosion, leading to an increase in the $\Omega$ parameter and indicating a greater influence of erosion on corrosion. This effect may be due to an increase in sand particle energy, as discussed by Wood ${ }^{6}$. Firstly, for the same impingement velocity, increasing particle size and particle mass increases the kinetic energy of sand particles, increasing the erosion rate and the depth/intensity of penetration/damage of the passive film, resulting in an increase in charge transfer at the liquid metal interface. Secondly, greater energy particles may plastically deform and stress the surface, enhancing corrosion processes and causing premature detachment of plastically deformed or strain hardened impact crater-lips. Thirdly, slurries composed by quartz particles with greater size could lead to unsteady hydrodynamics or turbulence leading to unstable double layers and unsteady driving concentration gradients of active species ${ }^{6}$.

\section{Conclusions}

The polarization curves give important information about the mechanism of corrosion-erosion under different tribological conditions. The following conclusions can be drawn:

1. The increase of the corrosion current density, measured by the reduction of the polarization resistance, could be related to the introduction of erosive particles in the flow.

2. Introducing a flow of electrolyte or erosive particles decreases the pitting potential. 
3. The introduction of bigger particles in the corrosive-erosive flow in $3.5 \% \mathrm{NaCl}$ solution led to a reduction of the polarization resistance of the surface in comparison with the reduction caused by the smaller particles. On the other hand, the change in the particle size did not show a marked effect on the pitting potential that remains low when particles are added to the flow.

4. There is no significant effect of impact angle on the electrochemical behaviour of AISI 304L in 3.5\% $\mathrm{NaCl}$ under the experimental conditions used in these tests.

5. The $\Omega$ parameter allows comparing the effect of the particle size on the electrochemical behaviour of the tested surfaces. As a consequence of the

\section{References}

1. Chen J, Zhang K, Li Q, Fu S, Wang SF. Corrosion and tribocorrosion behaviors of AISI 316 stainless steel and Ti6A14V alloys in artificial seawater. Tranactions of Nonferrous Metals Society of China. 2014;24(4):1022-1031. doi:10.1016/S10036326(14)63157-5

2. Aribo S, Barker R, Hu X, Neville A. Erosion-corrosion behaviour of lean duplex stainless steels in $3.5 \% \mathrm{NaCl}$ solution. Wear. 2013;302(1-2):1602-1608. DOI: 10.1016/j.wear.2012.12.007

3. Rajahram SS, Harvey TJ, Walker JC, Wang SC, Wood RJ. Investigation of erosion-corrosion mechanism of UNS S31603 using FIB and TEM. Tribology International 2012; 46(1):161-173.

4. Jones M, Llewellyn RJ. Erosion-corrosion assessment of materials for use in the resources industry. Wear. 2009;267(11):2003-2009. DOI: 10.1016/j.wear.2009.06.025

5. Hussain EA, Robinson MJ. Erosion-corrosion of 2205 duplex stainless steel in flowing seawater containing sand particles. Corrosion Science. 2007;49(4):1737-1754. DOI: 10.1016/j. corsci.2006.08.023

6. Wood RJ. Erosion-corrosion interactions and their effect on marine and offshore materials. Wear. 2006;261(9):1012-1023. doi:10.1016/j.wear.2006.03.033

7. Neville A, Hodgkiess T, Dallas JT. A study of the erosion-corrosion behaviour of engineering steels for marine pumping applications. Wear. 1995;186-187(Part 2):497-507. doi:10.1016/00431648(95)07145-8

8. Watson SW, Friedersdorf FJ, Madsen BW, Cramer SD. Methods of measuring wear-corrosion synergism. Wear. 1995;181-183(Part 2)::476-484. Doi:10.1016/0043-1648(95)90161-2

9. Madsen BW. Measurement of erosion-corrosion synergism with a slurry wear test apparatus. Wear. 1988;123(2):127-142. doi:10.1016/0043-1648(88)90095-6

10. Guenbour A, Faucheu J, Ben Bachir A, Dabosi F, Bui N. Electrochemical study of corrosion-abrasion of stainless steels in phosphoric acids. British Corrosion Journal. 1988;23(4):234238. DOI:10.1179/000705988798270631

11. Zhou S, Stack MM, Newman RC. Characterization of synergistic effects between erosion and corrosion in an aqueous environment addition of small size (50-75 AFS) particles, the passive current density increases almost twice, in comparison with the passive current obtained in liquid impingement. On the other hand, the addition of medium size (40-50 AFS) particles caused an increase in the passive current density of one order of magnitude while the introduction of big size particles (35-40 AFS) augmented the passive current density by a factor of 30 .

\section{Acknowledgements}

The authors acknowledge $\mathrm{CNPq}$ - Conselho Nacional de Desenvolvimento Científico e Tecnológico, Project n. 486104/2012-5 and FAPESP - Fundação de Amparo à Pesquisa do Estado de São Paulo, Project n. 2012/50890-0.

using electrochemical techniques. Corrosion. 1996;52(12):934946. http://dx.doi.org/10.5006/1.3292087

12. Burstein GT, Sasaki K. Detecting electrochemical transients generated by erosion corrosion. Electrochimica Acta. 2001;46(24):3675-3683. DOI: 10.1016/S0013-4686(01)00646-6

13. Wood RJ, Wharton JA, Speyer AJ, Tan KS. Investigation of erosion-corrosion processes using electrochemical noise measurements. Tribology International. 2002;35(10):631-641.

14. Ponthiaux P, Wenger F, Drees D, Celis JP. Electrochemical techniques for studying tribocorrosion processes. Wear. 2004;256(5):459-468. doi:10.1016/S0043-1648(03)00556-8

15. He DD, Jiang XX, Li S, Guan HR. Erosion-corrosion of stainless steels in aqueous slurries - a quantitative estimation of synergistic effects. Corrosion. 2005;61(1):30-36. http:// dx.doi.org/10.5006/1.3278157

16. Tait S. An introduction to electrochemical corrosion testing for practicing engineers and scientists. Racine Wisconsin: Pair O Docs; 1994.

17. Zheng Z, Zheng YG, Zhou X, He SY, Sun WH, Wang JQ. Determination of the critical flow velocities for erosioncorrosion of passive materials under impingement by $\mathrm{NaCl}$ solution containing sand. Corrosion Science. 2014;88:187-196. DOI: 10.1016/j.corsci.2014.07.043

18. Rajahram SS, Harvey TJ, Wood RJ. Electrochemical investigation of erosion-corrosion using a slurry pot erosion tester. Tribology International. 2011;44(3):232-240. doi:10.1016/j. triboint.2010.10.008

19. Lu BT, Mao LC, Luo JL. Hydrodynamic effects on erosion-enhanced corrosion of stainless steel in aqueous slurries. Electrochimica Acta. 2010;56(1):85-92. doi:10.1016/j.electacta.2010.09.047

20. Zhao Y, Zhou F, Yao J, Dong S, Ning L. Erosion-corrosion behavior and corrosion resistance of AISI 316 stainless steel in flow jet impingement. Wear. 2015;328-329:464-474. doi:10.1016/j.wear.2015.03.017

21. López D, Alonso-Falleiros N, Tschiptschin AP. Corrosionerosion behaviour of austenitic and martensitic high nitrogen stainless steels. Wear. 2007; 263(1):347-354. doi:10.1016/j. wear.2007.01.053

22. Fontana M, Greene N. Corrosion Engineering. New York: McGraw Hill; 1978. 465 p 